\title{
EDITORIAL
}

\section{Universal influenza vaccination in the elderly: another piece in the jigsaw}

\author{
Z. Borrill and M. Woodhead
}

I $\mathrm{t}$ is widely accepted that influenza causes significant mortality and morbidity in the elderly population during winter epidemics. Annual vaccination is recommended in the majority of western countries for all adults aged $>65$ yrs. Vaccination is aimed not at prevention of the spread of influenza, most of which occurs in children and younger adults, but at reduction of the complications among those who are most vulnerable, with death being the most significant, potentially preventable, complication. Each year, considerable resources are devoted to the creation of a vaccine against the influenza strains expected to be most prevalent, and to its timely administration. Despite the widespread endorsement of influenza vaccination for the elderly, its overall effectiveness continues to be debated.

Measuring the number of excess deaths attributable to influenza is difficult. Influenza-like illness (ILI) caused by other viruses is common and rarely distinguishable from influenza, due to a lack of virological conformation. Furthermore, influenza contributes to deaths from many causes which may not be recorded as such, the so called "hidden deaths". In developed countries, mortality is highest during the winter months, coinciding temporally with the existence of influenza epidemics. Studies without quantitative virological confirmation of influenza have estimated influenza deaths using seasonal mortality patterns and general information about influenza epidemic size. Whereas some argue that influenza is responsible for the majority of excess seasonal mortality [1], others conclude that its contribution is minimal [2].

Establishing the effectiveness of the influenza vaccine in improving mortality and morbidity has also been controversial. A randomised placebo-controlled trial demonstrated $>50 \%$ effectiveness in the prevention of serologically confirmed clinical influenza, but the study was not powered to determine the effect on mortality [3]. Justification of further placebo-controlled studies in the context of global recommendations for vaccination is ethically difficult. Furthermore, determination of vaccine efficacy (prevention of virologically proven influenza) in the ideal conditions of a randomised controlled trial may not necessarily translate to effectiveness

Dept of Respiratory Medicine, Manchester Royal Infirmary, Manchester, UK.

STATEMENT OF INTEREST: None declared.

CORRESPONDENCE: Z. Borrill, Dept of Respiratory Medicine, Manchester Royal Infirmary, Oxford Road, Manchester, M13 9WL, UK. Fax: 44 1612764989. E-mail: zoe@doctors.org.uk (prevention of clinical ILI) of a population vaccination programme. However, such uncontrolled observational studies are prone to selection bias, as those individuals seeking vaccination are likely to be different from those who abstain, the so-called "healthy-user effect". Furthermore, studies in selected cohorts may not be generalisable to the whole population. A recent systematic review of the effectiveness of influenza vaccination in the elderly showed that whereas in long-term care facilities vaccination significantly reduced influenza, ILI, pneumonia and death, the effectiveness in the community was modest [4]. Although vaccination reduced allcause mortality by $42 \%$ in the community, there was no significant reduction in influenza, ILI or pneumonia, providing evidence of possible selection bias. Whether, and to what extent, vaccination protects against death in the elderly remains a topic of debate [5].

In the current issue of the European Respiratory Journal, ÖRTQVIST et al. [6] present a comprehensive prospective cohort study assessing the effectiveness of the influenza vaccine on all-cause mortality in the elderly. The authors addressed the problem of selection bias by using influenza on- and off-season comparisons to compensate for differences between mortality observed in vaccinated and unvaccinated subjects, as well as adjusting for demographic factors and associated comorbidities. The study was carried out in Stockholm County (Sweden), which enabled the authors to collect comprehensive data on all adults aged $>65$ yrs during 3 consecutive years. Based on laboratory estimates, influenza activity during the first two seasons was moderate or high whereas there was no clear epidemic activity during the third season.

Vaccine effectiveness during the influenza season, adjusted for demographic factors, was similar for each season (44, 40 and $37 \%$, respectively) and comparable to that observed in other cohort studies. Furthermore, the authors observed a lower risk of death in vaccinated subjects during the influenza off-season, supporting previous claims of selection bias. After adjusting for this bias, a lower but nevertheless significant vaccine effectiveness of 14, 19 and $0 \%$ was observed during the three respective influenza seasons. ÖRTQVIST et al. [6] concluded that the number of elderly subjects needed to be vaccinated to prevent one death is estimated to be 150 to 300 during seasons of moderate-high epidemic levels.

Inclusion of all individuals in the population targeted by the vaccination is a major strength of this study. The other major difference between the study by ÖRTQVIST et al. [6] and 
previous studies is the attempt to correct for selection bias, not just by adjusting for demographic factors, which may not eliminate all potential confounders, but by comparing mortality data during the influenza on- and off-season. It remains possible that vaccinated individuals are more likely to die during the winter months than nonvaccinated individuals due to confounding factors other than infection with influenza and associated complications. Nevertheless, lack of a vaccination advantage during the third season where influenza epidemic activity was minimal suggests that the observed benefit in vaccination is caused by protection from influenza. Without virological confirmation linked to influenza related deaths, it is difficult to be certain. However, as vaccination can protect against severe forms of influenza and its complications without necessarily preventing all viral replication, such confirmation is difficult to achieve.

Evidence justifying the immense effort and cost of populationwide vaccination campaigns is drawn from many sources. This study provides a further piece in the jigsaw of supporting evidence for the benefits of the current policy of annual universal influenza vaccination in the elderly. In the event of a worldwide influenza pandemic, current seasonal vaccination strategies are unlikely to be of benefit until a strain-specific vaccination becomes available. However, such studies improve the evidence base available to policy makers planning the global strategy to confront this looming challenge.

\section{REFERENCES}

1 Reichert TA, Simonsen L, Sharma A, Pardo SA, Fedson DS, Miller MA. Influenza and the winter increase in mortality in the United States, 1959-1999. Am J Epidemiol 2004; 160: 492-502.

2 Donaldson GC, Keatinge WR. Excess winter mortality: influenza or cold stress? BMJ 2002; 324: 89-90.

3 Govaert TM, Thijs CT, Masurel N, Sprenger MJ, Dinant GJ, Knottnerus JA. The efficacy of influenza vaccination in elderly individuals. A randomized double-blind placebocontrolled trial. JAMA 1994; 272: 1661-1665.

4 Jefferson T, Rivetti D, Rivetti A, Rudin M, Di Pietrantonj C, Demicheli V. Efficacy and effectiveness of influenza vaccines in elderly people: a systematic review. Lancet 2005; 366: 1165-1174.

5 Jefferson T. Influenza vaccination: policy versus evidence. BMJ 2006; 333: 912-915.

6 Örtqvist A, Granath F, Askling J, Hedlund J. Influenza vaccination and mortality: prospective cohort study of the elderly in a large geographical area. Eur Respir J 2007; 30: 414-422. 